TITLE:

\title{
Fast multipole boundary element analysis for 2D problems of magneto-electro-elastic media
}

\section{$\operatorname{AUTHOR}(S)$ :}

Zhu, Xingyi; Huang, Zhiyi; Jiang, Aiming; Chen, W.Q.; Nishimura, N.

\section{CITATION:}

Zhu, Xingyi ...[et al]. Fast multipole boundary element analysis for 2D problems of magneto-electro-elastic media. Engineering Analysis with Boundary Elements 2010, 34(11): 927-933

\section{ISSUE DATE:}

2010-11

URL:

http://hdl.handle.net/2433/131751

\section{RIGHT:}

(C) 2010 Elsevier Ltd; This is not the published version. Please cite only the published version.; この論文は出版社版でありません。引用の際に は出版社版をご確認ご利用ください。 


\title{
Fast multipole boundary element analysis for $2 \mathrm{D}$ problems of magneto-electro-elastic media
}

\author{
Xingyi Zhu ${ }^{1}$, Zhiyi Huang ${ }^{1}$, Aiming Jiang ${ }^{2}$, W. Q. Chen ${ }^{3}$, N. Nishimura ${ }^{4, *}$ \\ ${ }^{1}$ Department of Civil Engineering, Zhejiang University, Zijingang Campus, Hangzhou 310058, P. R. China \\ ${ }^{2}$ Quzhou College, Quzhou 324006, Zhejiang, People's Republic of China \\ ${ }^{3}$ Department of Engineering Mechanics, Zhejiang University, Yuquan Campus, Hangzhou 310027, P. R. China \\ ${ }^{4}$ Department of Applied Analysis and Complex Dynamical Systems, Graduate School of Informatics, Kyoto \\ University, Kyoto 606-8501, Japan
}

\begin{abstract}
A two-dimensional (2D) fast multipole boundary element analysis of magneto-electro-elastic media has been developed in this paper. Fourier analysis is employed to derive the fundamental solution for the plane-strain magneto-electro-elasticity. The final formulations are very similar to those for the 2D potential problems, and hence it is quite easy to implement the fast multipole boundary element method. The results are verified by comparison with the analytical solutions to illustrate the accuracy and efficiency of the approach. The numerical examples of multi-inclusion magneto-electro-elastic composites are considered to show the versatility of the proposed approach in smart structure applications.
\end{abstract}

Key Words: Boundary element method, Fast multipole method, Fundamental solution, Magneto-electro-elastic media

\section{Introduction}

Magneto-electro-elastic (MEE) solids have wide technical applications, due to the mixed properties of the piezoelectric, piezomagnetic and magnetoelectric effects. This coupling behavior makes magneto-electro-elastic composite materials especially suitable for application in smart structures and micro-electromechanical systems (MEMS) or nano-electromechanical systems (NEMS).

Several approaches including numerical methods, analytical methods and experiments have been applied to the investigation of the coupling behavior of the MEE materials. Pan and coworkers [1,2] have pioneered analytical investigation of three-dimensional (3D) linear anisotropic, simply supported and multilayered MEE plates. Pan [3] further derived the 3D Green's functions in anisotropic MEE bimaterials. Wang and Shen [4] presented a general solution of the 3D equations of a transversely isotropic MEE medium, and derived the 3D Green's functions for a half-space and the exact solution for a generalized dislocation. Chen et al. [5,6] developed a new state-space formalism to study the static and dynamic behavior of multilayered or functionally graded MEE plates. Chen et al. [7] obtained a concise general solution for transversely isotropic MEE media involving thermal effect and derived an exact solution of a penny-shaped crack in an infinite body. Milazzo et al. [8] studied the forced vibration of a MEE

\footnotetext{
${ }^{*}$ Corresponding author. Tel.: +81 (75) 7535871 Fax: +81 (75) 7535924

Email address: nchml@i.kyoto-u.ac.jp
} 
bimorph beam based on the Timoshenko's beam theory.

A finite element method (FEM) analysis has been developed by Buchanan [9] to study the multilayer and multiphase MEE materials response. Petterman and Suresh [10] used a representative volume element to investigate the characteristics of 1-3 type piezo-composites along with the finite element technique. Lee et al. [11] obtained the effective properties of a three-phase MEE composite by employing the finite element method and found the non-zero magneto-electric coefficient. Besides finite element method, the boundary element method (BEM) can be regarded as an alternative feasible numerical method, due to its features of dimensionality reduction for linear problems and high accuracy. Ding and Jiang [12] derived the fundamental solution for the plane problem of MEE media in terms of harmonic functions and developed the boundary integral equations based on their fundamental solutions. Ding et al. [13] also presented 3D Green's functions for transversely isotropic MEE materials and performed 3D BEM analysis of an annular plate. By using a boundary element approach, Davi et al. [14] investigated the influence of the configuration of the magnetic layer on the behavior of MEE bimorph beams.

Fast multipole method (FMM) is an algorithm developed originally for numerical solution of integral equations (the reader is referred to the review article by Nishimura [15] for historical accounts, various applications and basic features of this method). It has been proved that the system of equations formed by BEM can be solved via FMM with higher efficiency and lower storage than with the traditional solvers. This method has been widely used in many areas of researches including electromechanical applications. For example, Sabariego et al. [16] recently applied a fast multipole method to the two-dimensional (2D) finite element-boundary element modeling of electromechanical devices, where a linear actuator is studied as an example application.

To develop a fast multipole method for BEM, one needs simple and appropriate expressions of fundamental solutions for 2D MEE problems. As mentioned above, researchers have already obtained various fundamental solutions for 2D MEE materials. However, their forms of solutions are somewhat complicated and in particular inappropriate to be implemented in the fast multipole method. Therefore, in this paper, we will first derive the fundamental solution by means of the Fourier transform. The expressions so obtained bear a similar structure as that of a $2 \mathrm{D}$ potential problem. Thus, it becomes very easy to program the 2D MEE counterpart based on available fast multipole BEM codes for 2D potential problems. Several numerical results are presented and verified by comparison with the analytical solutions to further illustrate the accuracy and efficiency of the approach. Furthermore, multiple inclusion problems are considered here to investigate the mixed properties of the piezoelectric, piezomagnetic and magnetoelectric effects. The effective properties of a piezoelectric-piezomagnetic composite are determined based on the numerical simulations. It is concluded that the method is not only easy in the meshing of complicated geometries, accurate for solving singular fields, but also practical in solving large-scale problems.

\section{Boundary integral equations for 2D MEE}

\subsection{Basic equations}

First, assume that the response of a magneto-electro-elastic body does not vary along the $y$ direction. This corresponds to a generalized plane-strain problem. With body force $\left(X_{1}, X_{2}\right)$, free electric charge $\left(X_{3}\right)$ and free magnetic charge $\left(X_{4}\right.$, which usually vanishes), the plane-strain 
governing field equations of MEE materials in the $x o z$ coordinates can be expressed by

$$
\begin{aligned}
& \frac{\partial \sigma_{x}}{\partial x}+\frac{\partial \tau_{x z}}{\partial z}+X_{1}=0, \frac{\partial \tau_{x z}}{\partial x}+\frac{\partial \sigma_{z}}{\partial z}+X_{2}=0 \\
& \frac{\partial D_{x}}{\partial x}+\frac{\partial D_{z}}{\partial z}-X_{3}=0, \frac{\partial B_{x}}{\partial x}+\frac{\partial B_{z}}{\partial z}-X_{4}=0
\end{aligned}
$$

where $\sigma_{i j}\left(\tau_{i j}\right)$ is the stress tensor, $D_{i}$ is the electric displacement vector, and $B_{i}$ is the magnetic induction vector. The 2D constitutive equations for a transversely isotropic MEE material are given by

$$
\begin{gathered}
\sigma_{x}=c_{11} \frac{\partial u}{\partial x}+c_{13} \frac{\partial w}{\partial z}+e_{31} \frac{\partial \Phi}{\partial z}+d_{31} \frac{\partial \Psi}{\partial z} \\
\tau_{x z}=c_{44}\left(\frac{\partial u}{\partial z}+\frac{\partial w}{\partial x}\right)+e_{15} \frac{\partial \Phi}{\partial x}+d_{15} \frac{\partial \Psi}{\partial x}, \sigma_{z}=c_{13} \frac{\partial u}{\partial x}+c_{33} \frac{\partial w}{\partial z}+e_{33} \frac{\partial \Phi}{\partial z}+d_{33} \frac{\partial \Psi}{\partial z} \\
-D_{x}=-e_{15}\left(\frac{\partial u}{\partial z}+\frac{\partial w}{\partial x}\right)+\varepsilon_{11} \frac{\partial \Phi}{\partial x}+g_{11} \frac{\partial \Psi}{\partial x} \\
-D_{z}=-e_{31} \frac{\partial u}{\partial x}-e_{33} \frac{\partial w}{\partial z}+\varepsilon_{33} \frac{\partial \Phi}{\partial z}+g_{33} \frac{\partial \Psi}{\partial z} \\
-B_{x}=-d_{15}\left(\frac{\partial u}{\partial z}+\frac{\partial w}{\partial x}\right)+g_{11} \frac{\partial \Phi}{\partial x}+\mu_{11} \frac{\partial \Psi}{\partial x} \\
-B_{z}=-d_{31} \frac{\partial u}{\partial x}-d_{33} \frac{\partial w}{\partial z}+g_{33} \frac{\partial \Phi}{\partial z}+\mu_{33} \frac{\partial \Psi}{\partial z}
\end{gathered}
$$

in which, $u, w$ are the displacement, $\Phi$ is the electric potential, and $\Psi$ is the magnetic potential. $c_{i j}, e_{i j}, d_{i j}, \varepsilon_{i j}, g_{i j}$ and $\mu_{i j}$ are the elastic, piezoelectric, piezomagnetic, dielectric, electromagnetic and magnetic constants, respectively.

The boundary conditions are given by

$$
\begin{gathered}
\sigma_{i j} n_{i}=\tilde{t}_{j} \text { on } \Gamma_{t} ; u_{i}=\tilde{u}_{i} \text { on } \Gamma_{u} \\
D_{i} n_{i}=-\widetilde{\omega} \text { on } \Gamma_{\omega} ; \Phi=\tilde{\phi} \text { on } \Gamma_{\phi} \\
B_{i} n_{i}=-\widetilde{\eta} \text { on } \Gamma_{\eta} ; \Psi=\tilde{\psi} \text { on } \Gamma_{\psi}
\end{gathered}
$$

where $t_{i}$ is the surface traction, $\omega$ is the surface charge, and $\eta$ is the surface magnetic induction. The tilde indicates the prescribed value, and $n_{i}$ denotes the unit outward normal vector.

Note that $\Gamma_{t}+\Gamma_{u}=\Gamma_{\omega}+\Gamma_{\phi}=\Gamma_{\eta}+\Gamma_{\psi}=\Gamma$, where $\Gamma$ is the whole boundary of the domain.

\subsection{Boundary integral formulations}

The boundary integral formulations for the 2D MEE media can be derived based on the 
Somigliana equation, and take the following form (See Appendix for derivation)

$$
\mathbf{C}(X) \mathbf{u}(X)=\int_{S} \mathbf{U}^{*}(X, \xi) \mathbf{t}(\xi) d \Gamma-\int_{\Gamma} \mathbf{T}^{*}(X, \xi) \mathbf{u}(\xi) d \Gamma+\int_{\Omega} \mathbf{U}^{*}(X, \xi) \mathbf{b}(\xi) d \Omega
$$

where $\mathbf{C}$ is the coefficient matrix, $\mathbf{U}^{*}$ and $\mathbf{T}^{*}$ are the generalized displacement and stress fundamental solutions of the 2D MEE problem, which will be discussed in the next section. The generalized displacement vector $\mathbf{u}$, the surface traction vector $\mathbf{t}$, and $\mathbf{U}^{*}$ and $\mathbf{T}^{*}$ are given by

$$
\mathbf{t}=\left\{\begin{array}{c}
t_{x} \\
t_{z} \\
-\omega \\
-\eta
\end{array}\right\}, \quad \mathbf{u}=\left\{\begin{array}{c}
u \\
w \\
-\phi \\
-\psi
\end{array}\right\}, \quad \mathbf{U}^{*}=\left\{\begin{array}{llll}
u_{11}^{*} & u_{12}^{*} & \phi_{1}^{*} & \psi_{1}^{*} \\
u_{21}^{*} & u_{22}^{*} & \phi_{2}^{*} & \psi_{2}^{*} \\
u_{31}^{*} & u_{32}^{*} & \phi_{3}^{*} & \psi_{3}^{*} \\
u_{41}^{*} & u_{42}^{*} & \phi_{4}^{*} & \psi_{4}^{*}
\end{array}\right\}, \mathbf{T}^{*}=\left\{\begin{array}{cccc}
t_{11}^{*} & t_{12}^{*} & \omega_{1}^{*} & \eta_{1}^{*} \\
t_{21}^{*} & t_{22}^{*} & \omega_{2}^{*} & \eta_{2}^{*} \\
t_{31}^{*} & t_{32}^{*} & \omega_{3}^{*} & \eta_{3}^{*} \\
t_{41}^{*} & t_{42}^{*} & \omega_{4}^{*} & \eta_{4}^{*}
\end{array}\right\}
$$

where $u_{i j}^{*}$ and $t_{i j}^{*}(i, j=1,2)$ denote the displacement and traction components in the $j$-th direction at a point $\xi$ due to a unit force acting in the $i$-th direction at the point $X ; u_{3 j}^{*}$ and $t_{3 j}^{*}(j=1,2)$ represent the $j$-th displacement and traction at $\xi$ due to a unit electric charge at $X ; u_{4 j}^{*}$ and $t_{4 j}^{*}$ $(j=1,2)$ represent the $j$-th displacement and traction at $\xi$ due to a unit magnetic charge at $X ; \phi_{i}^{*}$, $\psi_{i}^{*}, \omega_{i}^{*}, \eta_{i}^{*}(i=1,2)$ stand for the electric potential, magnetic potential, the surface charge and surface magnetic induction at $\xi$ due to a unit force acting in the $i$-th direction at $X ; \phi_{3}^{*}, \psi_{3}^{*}, \omega_{3}^{*}$, $\eta_{3}^{*}$ stand for the electric potential, magnetic potential, the surface charge and surface magnetic induction at $\xi$ due to a unit electric charge at $X ; \phi_{4}^{*}, \psi_{4}^{*}, \omega_{4}^{*}, \eta_{4}^{*}$ stand for the electric potential, magnetic potential, the surface charge and surface magnetic induction at $\xi$ due to a unit magnetic charge at $X$.

\section{2D fast multipole formulations for MEE media}

First, substituting Eq. (2) into Eq. (1), we can obtain the basic equations of the 2D magneto-electro-elasticity as

$$
D\left\{\begin{array}{l}
U \\
W \\
\Phi \\
\Psi
\end{array}\right\}+\left\{\begin{array}{c}
X_{1} \\
X_{2} \\
-X_{3} \\
-X_{4}
\end{array}\right\}=\mathbf{0}
$$

where $D$ is the following differential operator matrix 


$$
D=\left[\begin{array}{cccc}
c_{11} \partial_{x x}+c_{44} \partial_{z z} & \left(c_{13}+c_{44}\right) \partial_{x z} & \left(e_{15}+e_{31}\right) \partial_{x z} & \left(d_{15}+d_{31}\right) \partial_{x z} \\
\left(c_{13}+c_{44}\right) \partial_{x z} & c_{44} \partial_{x x}+c_{33} \partial_{z z} & e_{15} \partial_{x x}+e_{33} \partial_{z z} & d_{15} \partial_{x x}+d_{33} \partial_{z z} \\
\left(e_{15}+e_{31}\right) \partial_{x z} & e_{15} \partial_{x x}+e_{33} \partial_{z z} & -\left(\varepsilon_{11} \partial_{x x}+\varepsilon_{33} \partial_{z z}\right) & -\left(g_{11} \partial_{x x}+g_{33} \partial_{z z}\right) \\
\left(d_{15}+d_{31}\right) \partial_{x z} & d_{15} \partial_{x x}+d_{33} \partial_{z z} & -\left(g_{11} \partial_{x x}+g_{33} \partial_{z z}\right) & -\left(\mu_{11} \partial_{x x}+\mu_{33} \partial_{z z}\right)
\end{array}\right]
$$

in which, $\partial_{x x}=\frac{\partial^{2}}{\partial x^{2}}, \partial_{x z}=\frac{\partial^{2}}{\partial x \partial z}$, and $\partial_{z z}=\frac{\partial^{2}}{\partial z^{2}}$. To obtain a proper form of the fundamental solution of the above equations, we here use Fourier transform defined by

$$
\begin{gathered}
\hat{F}\left(\lambda_{1}, \lambda_{2}\right)=\int_{-\infty}^{\infty} \int_{-\infty}^{\infty} F(x, z) e^{-i\left(\lambda_{1} x+\lambda_{2} z\right)} d x d z \\
F(x, z)=\left(\frac{1}{2 \pi}\right)^{2} \int_{-\infty}^{\infty} \int_{-\infty}^{\infty} \hat{F}\left(\lambda_{1}, \lambda_{2}\right) e^{i\left(\lambda_{1} x+\lambda_{2} z\right)} d \lambda_{1} d \lambda_{2}
\end{gathered}
$$

where $i=\sqrt{-1}$. Thus, Eq. (6) can be written in the transform domain as follows,

$$
\left\{\begin{array}{l}
\hat{U} \\
\hat{W} \\
\hat{\Phi} \\
\hat{\Psi}
\end{array}\right\}=\left\{\begin{array}{llll}
A_{11} & A_{12} & A_{13} & A_{14} \\
A_{12} & A_{22} & A_{23} & A_{24} \\
A_{13} & A_{23} & A_{33} & A_{34} \\
A_{14} & A_{24} & A_{34} & A_{44}
\end{array}\right\}\left\{\begin{array}{c}
\hat{X}_{1} \\
\hat{X}_{2} \\
-\hat{X}_{3} \\
-\hat{X}_{4}
\end{array}\right\}
$$

where $\hat{X}_{1}$ and $\hat{X}_{2}$ are body forces in transform domain, $\hat{X}_{3}$ and $\hat{X}_{4}$ are free electric charge and free magnetic charge in transform domain respectively. Also, $A_{k l}=-\frac{P_{k l}\left(\lambda_{1}, \lambda_{2}\right)}{|\hat{D}|}, P_{k l}$ is the cofactors of $\hat{D}, \hat{D}$ is the Fourier transform of $D$, and $|\hat{D}|$ is the determinant of the matrix $\hat{D}$, which can be evaluated as

$$
|\hat{D}|=a_{1} \lambda_{2}^{8}+a_{2} \lambda_{2}^{6} \lambda_{1}^{2}+a_{3} \lambda_{1}^{4} \lambda_{2}^{4}+a_{4} \lambda_{1}^{6} \lambda_{2}^{2}+a_{5} \lambda_{1}^{8}
$$

where the coefficients $a_{n}(n=1,2, \ldots, 5)$ are the same as those given by Hou et al. [17]. Eq. (10) can be decomposed into four factors as

$$
|\hat{D}|=a_{5}\left(\lambda_{1}^{2}+\lambda_{2}^{2} / s_{1}^{2}\right)\left(\lambda_{1}^{2}+\lambda_{2}^{2} / s_{2}^{2}\right)\left(\lambda_{1}^{2}+\lambda_{2}^{2} / s_{3}^{2}\right)\left(\lambda_{1}^{2}+\lambda_{2}^{2} / s_{4}^{2}\right)
$$

where $s_{j}(j=1,2,3,4)$ are the four roots of the following algebraic equation which satisfy $\operatorname{Re}\left(s_{j}\right)>0$ :

$$
a_{1} s^{8}-a_{2} s^{6}+a_{3} s^{4}-a_{4} s^{2}+a_{5}=0
$$

These roots $s_{j}$ are related to the material constants only. Here we confine ourselves to the simple but 
frequently encountered case that all these roots are distinct.

Now, the Fourier inverse transform of Eq. (9) shall give us the fundamental solution in the physical space. Before performing the inversion, however, we rewrite the fundamental solution in the following form to conform to the boundary integral formulation in Eq. (5). For example,

$$
\begin{aligned}
u_{k l}^{*} & =\frac{1}{2 \pi} \frac{1}{2 \pi} \int_{-\infty}^{\infty} \int_{-\infty}^{\infty} A_{k l} e^{i\left(\lambda_{1} x+\lambda_{2} z\right)} d \lambda_{1} d \lambda_{2} \\
& =\frac{1}{2 \pi} \frac{1}{2 \pi} \int_{-\infty}^{\infty} \int_{-\infty}^{\infty} \frac{P_{k l}^{s}\left(\lambda_{1}, \lambda_{2}\right) e^{i\left(\lambda_{1} x+\lambda_{2} z\right)} d \lambda_{1} d \lambda_{2}}{\left(s_{1}^{2} \lambda_{1}^{2}+\lambda_{2}^{2}\right)\left(s_{2}^{2} \lambda_{1}^{2}+\lambda_{2}^{2}\right)\left(s_{3}^{2} \lambda_{1}^{2}+\lambda_{2}^{2}\right)\left(s_{4}^{2} \lambda_{1}^{2}+\lambda_{2}^{2}\right)}
\end{aligned}
$$

where

$$
P_{k l}^{s}\left(\lambda_{1}, \lambda_{2}\right)=-\frac{s_{1}^{2} s_{2}^{2} s_{3}^{2} s_{4}^{2}}{a_{5}} P_{k l}\left(\lambda_{1}, \lambda_{2}\right)
$$

By using the residue theorem, we can obtain from Eq. (13):

$$
u_{k l}^{*}=\frac{i}{2 \pi} \sum_{m=1}^{4} \int_{-\infty}^{\infty} \frac{P_{k l}^{s}\left(\lambda_{1}, s_{m} i\left|\lambda_{1}\right|\right) e^{-s_{m}\left|\lambda_{1}\right| z+i \lambda_{1} x}}{2 s_{m} b_{m} i\left|\lambda_{1}\right| \lambda_{1}^{6}} d \lambda_{1}
$$

for a positive $z$, where

$$
\begin{aligned}
& b_{1}=\left(s_{2}^{2}-s_{1}^{2}\right)\left(s_{3}^{2}-s_{1}^{2}\right)\left(s_{4}^{2}-s_{1}^{2}\right) \\
& b_{2}=\left(s_{1}^{2}-s_{2}^{2}\right)\left(s_{3}^{2}-s_{2}^{2}\right)\left(s_{4}^{2}-s_{2}^{2}\right) \\
& b_{3}=\left(s_{1}^{2}-s_{3}^{2}\right)\left(s_{2}^{2}-s_{3}^{2}\right)\left(s_{4}^{2}-s_{3}^{2}\right) \\
& b_{4}=\left(s_{1}^{2}-s_{4}^{2}\right)\left(s_{2}^{2}-s_{4}^{2}\right)\left(s_{3}^{2}-s_{4}^{2}\right)
\end{aligned}
$$

Since $P_{k l}^{s}$ is a polynomial of sixth order about $\lambda_{1}$, we can get the following equation from Eq.(15) as

$$
u_{k l}^{*}=\frac{1}{4 \pi} \sum_{m=1}^{4}\left[\frac{P_{k l}^{s}\left(-1, s_{m} i\right)}{s_{m} b_{m}} \int_{0}^{\infty} \frac{e^{-i \lambda_{1} x-s_{m} \lambda_{1} z}}{\lambda_{1}} d \lambda_{1}+\frac{P_{k l}^{s}\left(1, s_{m} i\right)}{s_{m} b_{m}} \int_{0}^{\infty} \frac{e^{i \lambda_{1} x-s_{m} \lambda_{1} z}}{\lambda_{1}} d \lambda_{1}\right]
$$

where the two integrals are computed in the sense of finite parts. Therefore, we can obtain

$$
u_{k l}^{*}=\frac{1}{4 \pi} \sum_{m=1}^{4}\left[\frac{P_{k l}^{s}\left(-1, s_{m} i\right)}{s_{m} b_{m}} \ln \left(x-i s_{m} z\right)+\frac{P_{k l}^{s}\left(1, s_{m} i\right)}{s_{m} b_{m}} \ln \left(x+i s_{m} z\right)\right]
$$

to within a non-essential additive constant. This result can be further rewritten as

$$
\begin{gathered}
u_{k l}^{*}=\frac{1}{4 \pi} \sum_{m=1}^{4}\left[M_{k l}^{m} \ln \left(x+i s_{m} z\right)+\overline{M_{k l}^{m} \ln \left(x+i s_{m} z\right)}\right] \\
M_{k l}^{m}=\frac{P_{k l}^{s}\left(1, s_{m} i\right)}{s_{m} b_{m}}
\end{gathered}
$$


in which the overbar means the complex conjugate of the variable. The cofactors $P_{k l}\left(\lambda_{1}, \lambda_{2}\right)$ of $\hat{D}$ can be easily calculated; for example, we have

$$
\begin{aligned}
& P_{21}\left(\lambda_{1}, \lambda_{2}\right)=-n_{1} \lambda_{1}^{5} \lambda_{2}-n_{2} \lambda_{1}^{3} \lambda_{2}^{3}-n_{3} \lambda_{1} \lambda_{2}{ }^{5} \\
& P_{22}\left(\lambda_{1}, \lambda_{2}\right)=n_{41} \lambda_{1}{ }^{6}+n_{51} \lambda_{1}{ }^{4} \lambda_{2}{ }^{2}+n_{61} \lambda_{1}{ }^{2} \lambda_{2}{ }^{4}+n_{71} \lambda_{2}{ }^{6} \\
& P_{23}\left(\lambda_{1}, \lambda_{2}\right)=n_{42} \lambda_{1}{ }^{6}+n_{52} \lambda_{1}{ }^{4} \lambda_{2}{ }^{2}+n_{62} \lambda_{1}{ }^{2} \lambda_{2}{ }^{4}+n_{72} \lambda_{2}{ }^{6} \\
& P_{24}\left(\lambda_{1}, \lambda_{2}\right)=n_{43} \lambda_{1}{ }^{6}+n_{53} \lambda_{1}{ }^{4} \lambda_{2}{ }^{2}+n_{63} \lambda_{1}{ }^{2} \lambda_{2}{ }^{4}+n_{73} \lambda_{2}{ }^{6}
\end{aligned}
$$

where $n_{k}(k=1,2,3)$ and $n_{k l}(k=4,5,6,7 ; l=1,2,3)$ can be found in Ref. [12]. Other cofactors of $P_{k l}\left(\lambda_{1}, \lambda_{2}\right)$ can be obtained similarly. We note that the result in Eq.(19) remains valid for a negative $z$.

To further get the kernel function of $t_{k l}^{*}$, one just substitutes the fundamental solution in Eq. (19) into the constitutive relations. This leads to the following expressions of the surface tractions

$$
\left\{\begin{array}{c}
t_{k 1} \\
t_{k 2} \\
t_{k 3} \\
t_{k 4}
\end{array}\right\}=\frac{1}{4 \pi} \sum_{m=1}^{4} \mathbf{B} \cdot\left(\frac{1}{x+i s_{m} z} \mathbf{M S}_{m}+\frac{1}{x+i s_{m} z} \mathbf{M S}_{m}\right), \quad(k=1,2,3,4)
$$

where

$$
\begin{gathered}
\mathbf{M S}_{m}=\left[\begin{array}{lllllllll}
M_{k 1}^{m} & i s_{m} M_{k 1}^{m} & M_{k 2}^{m} & i s_{m} M_{k 2}^{m} & M_{k 3}^{m} & i s_{m} M_{k 3}^{m} & M_{k 4}^{m} & i s_{m} M_{k 4}^{m}
\end{array}\right]^{T} \\
\mathbf{B}=\left[\begin{array}{lllllllll}
c_{11} n_{x} & c_{44} n_{z} & c_{44} n_{z} & c_{13} n_{x} & e_{15} n_{z} & e_{31} n_{x} & d_{15} n_{z} & d_{31} n_{x} \\
c_{13} n_{z} & c_{44} n_{x} & c_{44} n_{x} & c_{33} n_{z} & e_{15} n_{x} & e_{33} n_{z} & d_{15} n_{x} & d_{33} n_{z} \\
e_{31} n_{z} & e_{15} n_{x} & e_{15} n_{x} & e_{33} n_{z} & -\varepsilon_{11} n_{x} & -\varepsilon_{33} n_{z} & -g_{11} n_{x} & -g_{33} n_{z} \\
d_{31} n_{z} & d_{15} n_{x} & d_{15} n_{x} & d_{33} n_{z} & -g_{11} n_{x} & -g_{33} n_{z} & -\mu_{11} n_{x} & -\mu_{33} n_{z}
\end{array}\right]
\end{gathered}
$$

Hence, we have obtained the 2D fundamental solution of magnetic-electric-elastic media. These solutions are at least as simple as the existing ones. However, it is much easier to develop FMM-BEM algorithms based on the present fundamental solutions, as shown below.

From the above fundamental solutions, we find the following basic function when the loading is acted at any point,

$$
G_{j}\left(z_{j}^{0}, z_{j}\right)=\ln \left(z_{j}^{0}-z_{j}\right) \quad(j=1,2,3,4)
$$

For convenience, we introduce the complex notation, $z_{j}^{0}=x+i z s_{j}, z_{j}=\xi+i \zeta s_{j}$, with the point $(x$, 
$z$ ) being the observation point, and the point $(\xi, \zeta)$ being the field point or integration point. Thus, the basic equation is almost the same as the fundamental solution of a 2D potential problem, except for the existence of $s_{j}$, which may be a complex number according to the material properties. Nevertheless, the general idea of the multipole expansions of this function is totally the same as the 2D potential problem. For example, the basic function can be expanded as follows,

$$
\int_{\Gamma_{j}^{0}} G_{j}\left(z_{j}^{0}, z_{j}\right) t\left(z_{j}\right) d \Gamma\left(z_{j}\right)=\sum_{k=0}^{\infty} O_{k}\left(z_{j}^{0}-z_{j}^{c}\right) H_{k}\left(z_{j}^{c}\right) \quad(j=1,2,3,4)
$$

where

$$
H_{k}\left(z_{j}^{c}\right)=\int_{\Gamma_{j}^{0}} I_{k}\left(z_{j}-z_{j}^{c}\right) t\left(z_{j}\right) d \Gamma\left(z_{j}\right)
$$

is called the moment about the mid point $z_{j}^{c}$, and

$$
I_{k}(z)=\frac{z^{k}}{k !}, \text { for } k \geq 0 ; O_{k}(z)=\frac{(k-1) !}{z^{k}}, \text { for } k \geq 1 ; O_{0}(z)=\ln (z)
$$

The mid point $z_{j}^{c}$ is close to $\Gamma_{j}^{0}$ (i.e. $\left|z_{j}-z_{j}^{c}\right|<<\left|z_{j}^{0}-z_{j}^{c}\right|$, for $z_{j} \in \Gamma_{j}^{0}$ ), and can be expressed by $z_{j}^{c}=\xi_{c}+i \zeta_{c} s_{j}$

It should be mentioned that $\Gamma_{j}^{0}$ is no longer the original boundary of the whole body. Indeed, we have four different boundaries according to the assumption of $s_{j}$. A simple way to deal with this situation is to introduce four tree structures, which depend on the different boundaries $\Gamma_{j}^{0}$ of the body. Then, it will become very convenient to implement Eqs. (19), (21) and (23) in the FMM algorithm based on these four tree structures. It is also understandable that a shift of multipole expansion (M2M), a shift of multipole to local expansion (M2L) and a shift of local expansion (L2L) remain almost the same as in the potential problem. Detailed analysis and formulations of the subsequent multipole translations and implementations of FMM can be found in Ref. [18].

We note that the formulation given above leads to an $O(N)$ numerical method in problems with $N$ unknowns because it basically invokes the FMM for Laplace's equation (an well-established $O(N)$ method) 4 times for each evaluation of the integrals in Eq.(4) on the whole boundary.

\section{Numerical Examples}

Several examples are considered here to verify the proposed numerical procedures of the fast multipole BEM for 2D magneto-electro-elastic problems. In the following examples, constant elements are employed to discretize the boundary of the domain, the numbers of terms for multipole and local expansions are set to 20 , the number of elements allowed in a leaf is 20 , and the tolerance for convergence in the iterative solver is $10^{-6}$.

\subsection{A plate under prescribed tension}


Consider a magneto-electro-elastic rectangle of size $a \times b$ under the following boundary conditions, see Fig.1,

$$
\begin{aligned}
& z=+b / 2: t_{x}=0, t_{z}=100, \omega=0, \eta=0 \\
& z=-b / 2: t_{x}=0, w=0, \phi=0, \psi=0 \\
& x=+a / 2: t_{x}=0, t_{z}=0, \omega=0, \eta=0 \\
& x=-a / 2: u=0, t_{z}=0, \omega=0, \eta=0
\end{aligned}
$$

The plane-strain state is assumed. Table 1 presents the material constants used in the calculation.

Table 1. Material constants

\begin{tabular}{|c|c|c|c|c|c|c|c|}
\hline$c_{11}$ & $c_{13}$ & $c_{33}$ & $c_{44}$ & $e_{31}$ & $e_{33}$ & $e_{15}$ & $\varepsilon_{11}$ \\
\hline $1.66 \mathrm{E} 11$ & $7.8 \mathrm{E} 10$ & $1.62 \mathrm{E} 11$ & $4.3 \mathrm{E} 10$ & -4.4 & 18.6 & 11.6 & $11.2 \mathrm{E}-9$ \\
\hline$\varepsilon_{33}$ & $d_{31}$ & $d_{33}$ & $d_{15}$ & $g_{11}$ & $g_{33}$ & $\mu_{11}$ & $\mu_{33}$ \\
\hline $12.6 \mathrm{E}-9$ & 580.3 & 699.7 & 550 & $5 \mathrm{E}-12$ & $3 \mathrm{E}-12$ & $5 \mathrm{E}-6$ & $10 \mathrm{E}-6$ \\
\hline
\end{tabular}

Unit: $C$ - $\mathrm{N} / \mathrm{m}^{2}, e-\mathrm{C} / \mathrm{m}^{2}, d-\mathrm{N} / \mathrm{Am}, \varepsilon-\mathrm{C} / \mathrm{Vm}, \mu-\mathrm{Ns}^{2} / \mathrm{C}^{2}, \quad g-\mathrm{Ns} / \mathrm{VC}$.

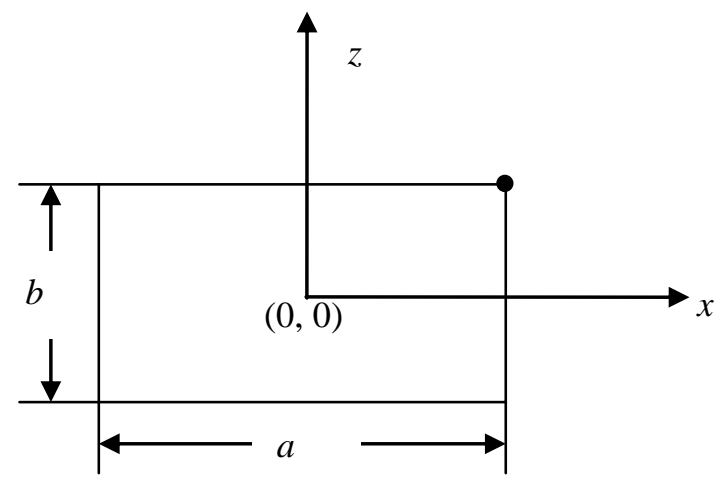

Fig. 1 Simple tension of a magneto-electro-elastic media

For numerical calculation, we consider the rectangle of $a=2.0 \mathrm{~m}$ and $b=2.0 \mathrm{~m}$ for which the total number of elements on the boundary ranges from 16 to 5120. Table 2 shows the results of the displacement, electric potential and magnetic potential at the point $(a / 2, b / 2)$. With only 80 elements on the boundary, the results are already very accurate and stable by the fast multipole boundary element method. Compared with the analytical solutions, the maximum error is less than $3 \%$.

Table 2. Comparison of FMBEM results with the exact solution

\begin{tabular}{|c|c|c|c|c|c|}
\hline $\mathrm{N}$ & DOFs & $u(\times 1 . \mathrm{E} 10)$ & $w(\times 1 . \mathrm{E} 10)$ & $\phi$ & $\psi$ \\
\hline 16 & 64 & $-0.567616 \mathrm{E}+01$ & $1.156720 \mathrm{E}+01$ & $1.880028 \mathrm{E}+00$ & $0.408640 \mathrm{E}-01$ \\
\hline 80 & 320 & $-0.619428 \mathrm{E}+01$ & $1.138348 \mathrm{E}+01$ & $1.904264 \mathrm{E}+00$ & $0.425132 \mathrm{E}-01$ \\
\hline
\end{tabular}




\begin{tabular}{|c|c|c|c|c|c|}
\hline 160 & 640 & $-0.626124 \mathrm{E}+01$ & $1.136984 \mathrm{E}+01$ & $1.905884 \mathrm{E}+00$ & $0.426592 \mathrm{E}-01$ \\
\hline 320 & 1,280 & $-0.629592 \mathrm{E}+01$ & $1.136572 \mathrm{E}+01$ & $1.906428 \mathrm{E}+00$ & $0.427220 \mathrm{E}-01$ \\
\hline 640 & 2,560 & $-0.631400 \mathrm{E}+01$ & $1.136512 \mathrm{E}+01$ & $1.906592 \mathrm{E}+00$ & $0.427488 \mathrm{E}-01$ \\
\hline 1,280 & 5,120 & $-0.632352 \mathrm{E}+01$ & $1.136564 \mathrm{E}+01$ & $1.906628 \mathrm{E}+00$ & $0.427604 \mathrm{E}-01$ \\
\hline 2,560 & 10,240 & $-0.632848 \mathrm{E}+01$ & $1.136636 \mathrm{E}+01$ & $1.906632 \mathrm{E}+00$ & $0.427652 \mathrm{E}-01$ \\
\hline 5,120 & 20,480 & $-0.633152 \mathrm{E}+01$ & $1.136628 \mathrm{E}+01$ & $1.906692 \mathrm{E}+00$ & $0.427744 \mathrm{E}-01$ \\
\hline \multicolumn{2}{|r|}{ nnalytical solution } & $-0.633316 \mathrm{E}+01$ & $1.136676 \mathrm{E}+01$ & $1.899100 \mathrm{E}+00$ & $0.427812 \mathrm{E}-01$ \\
\hline
\end{tabular}

The CPU time for the FMBEM to solve the models with 5120 DOFs, for example, was 692s, while that for the conventional BEM with direct solver was about 7810s, thus showing the advantage of the proposed method over the conventional one. We note, however, that these timing results are just rough indications of the relative efficiencies, because they are obtained on a laptop computer (Intel Core2 CPU) with limited resources.

\subsection{A single-inclusion problem}

Composite materials consisting of both piezoelectric phase and piezomagnetic phase have broad applications in the fabrication of smart and adaptive material systems, due to the mixed properties of the piezoelectric, piezomagnetic and magnetoelectric effects. Here, we will study the coupling behavior of heterogeneous magneto-electro-elastic materials with an embedded inclusion to verify the efficiency of the new fast multipole BEM for 2D multi-domain MEE problems.

We consider a square sheet with one inclusion, see Fig. 2. The inclusion has the same material constants as the matrix, as shown in Table 1. To obtain the effective material properties of the magneto-electro-elastic sheet, one should impose a series of special loads with different boundary conditions. For example, all the field variables except the strain in the $x$-direction will vanish identically when the sheet is subjected to a uniform unit displacement along the $x$-axis direction at the edge $\mathrm{AB}$, while the normal displacements and tangential tractions are zero along the edges $\mathrm{AD}$, $\mathrm{CD}$ and $\mathrm{BC}$ and the electric potentials and the magnetic potentials are set zero at all the four edges. Then, it is easy to get the effective material constants $c_{11}$ and $c_{13}$ according to this special kind of boundary conditions.

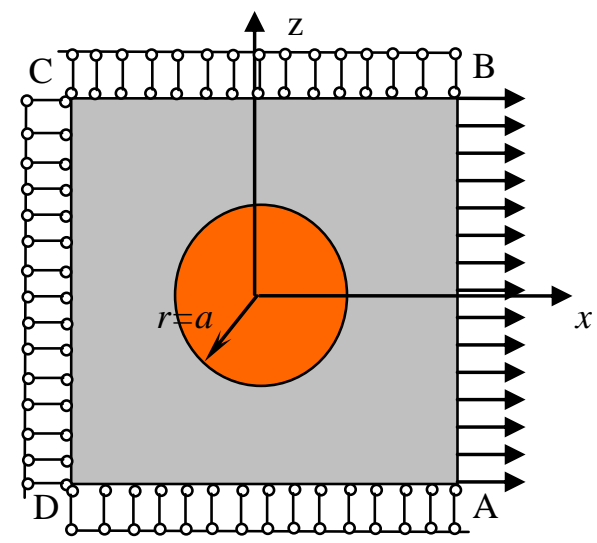

Fig. 2 A single-inclusion model 
Since the matrix and the inclusion have the same material properties, we will get the same effective material constants as those listed in the Table 1 after performing the fast multipole BEM calculation. Table 3 presents the results predicted by FMBEM with $200 \times n$ elements on the outer boundary and $200 \times n$ elements on the interface. This example clearly demonstrates the accuracy and efficiency of the fast multipole BEM for solving multi-domain 2D MEE problems.

Table 3. Computed effective material constants (the units are the same as in Table 1).

\begin{tabular}{|c|c|c|c|c|c|c|}
\hline$n$ & $c_{11}$ & $c_{13}$ & $e_{31}$ & $e_{33}$ & $d_{33}$ & $d_{31}$ \\
\hline 1 & $1.667 \mathrm{E}+11$ & $7.83 \mathrm{E}+10$ & -4.21 & 18.75 & 706.6 & 583.2 \\
\hline 2 & $1.663 \mathrm{E}+11$ & $7.82 \mathrm{E}+10$ & -4.34 & 18.67 & 702.1 & 582.3 \\
\hline 3 & $1.661 \mathrm{E}+11$ & $7.81 \mathrm{E}+10$ & -4.37 & 18.66 & 700.8 & 581.8 \\
\hline 4 & $1.661 \mathrm{E}+11$ & $7.80 \mathrm{E}+10$ & -4.38 & 18.63 & 700.3 & 580.9 \\
\hline 5 & $1.660 \mathrm{E}+11$ & $7.80 \mathrm{E}+10$ & -4.39 & 18.61 & 700.0 & 580.5 \\
\hline exact & $1.66 \mathrm{E}+11$ & $7.8 \mathrm{E}+10$ & -4.4 & 18.6 & 699.7 & 580.3 \\
\hline
\end{tabular}

\subsection{Multi-inclusion composite}

We now consider a piezoelectric-piezomagnetic composite, in which the circular inclusions of piezoelectric phase $\left(\mathrm{BaTiO}_{3}\right)$ are embedded in the matrix of piezomagnetic phase $\left(\mathrm{CoFe}_{2} \mathrm{O}_{4}\right)$, see Fig. 3. The interface between each inclusion and the matrix is perfect, and the material constants of $\mathrm{BaTiO}_{3}$ and $\mathrm{CoFe}_{2} \mathrm{O}_{4}$ can be found in Ref.[19]. In our models, the number of inclusions is kept to be 100 , and the radius of inclusions is changed with the volume fraction of the inclusion $V_{f .} 400$ elements are used on the outer boundary of the square sheet, and 80 elements are used for each inclusion.

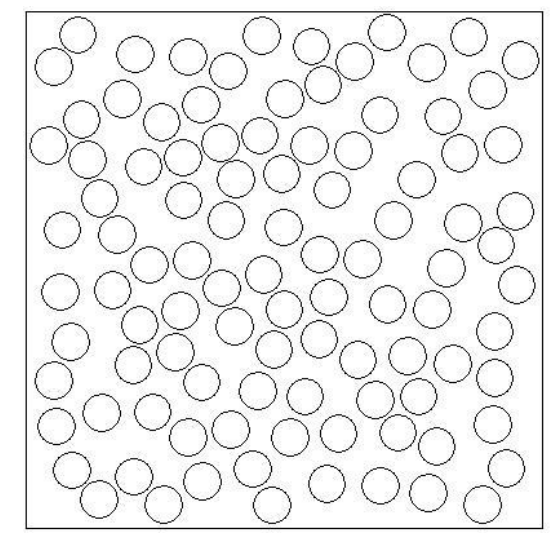

Fig. 3 A piezoelectric-piezomagnetic composite model

The results obtained from the FMBEM simulation and analytical solution (based on the simple rule of mixture [20]) are plotted in Figs.4-6. Three values of the volume fraction $V_{f}$ (i.e. 0.1, 0.3 and 0.5) are considered here for the pizeoelectric inclusions. Although the fast multipole BEM predictions are a little lower than the analytical solution, they are basically in good agreement for all material constants presented in these figures, which show the effects of $V_{f}$ on the effective elastic moduli, effective piezoelectric constants and effective piezomagnetic constants, respectively. 
It is seen for a magneto-electro-elastic composite reinforced by the piezoelectric phase $\left(\mathrm{BaTiO}_{3}\right)$, that the effective elastic modulus and the effective piezomagnetic constants will decrease with $V_{f}$.

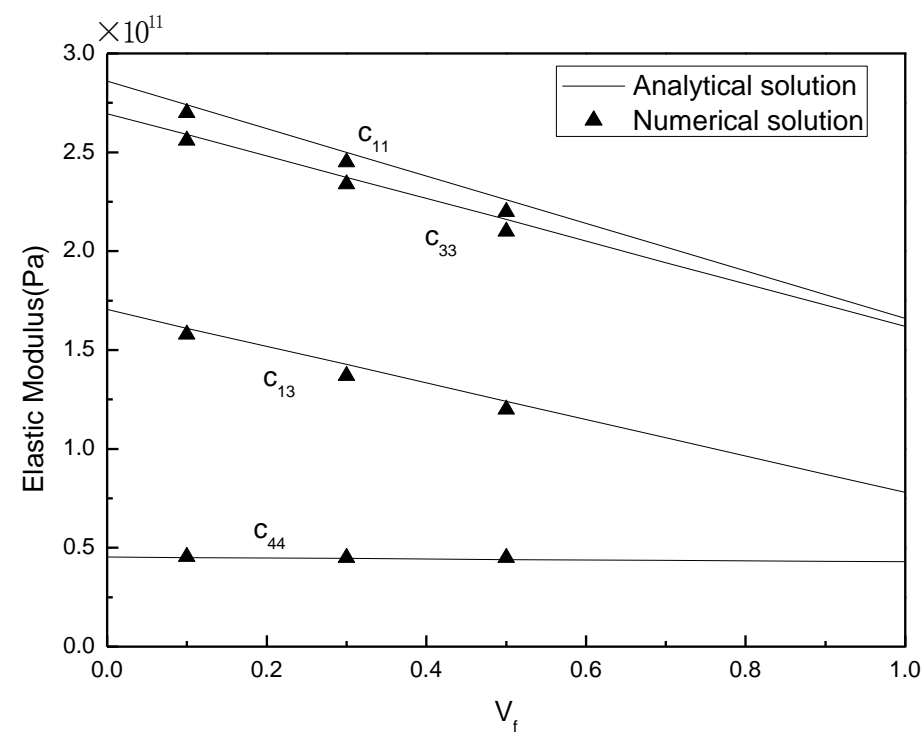

Fig. 4 The effects of volume fraction on the effective elastic constants

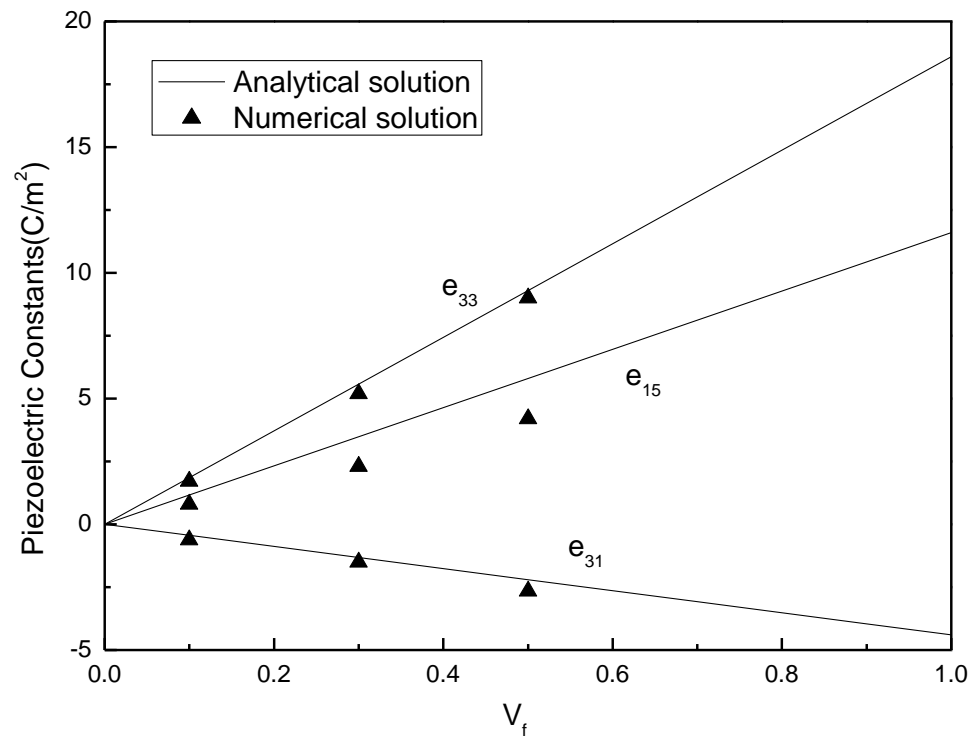

Fig. 5 The effects of volume fraction on the effective piezoelectric constants 


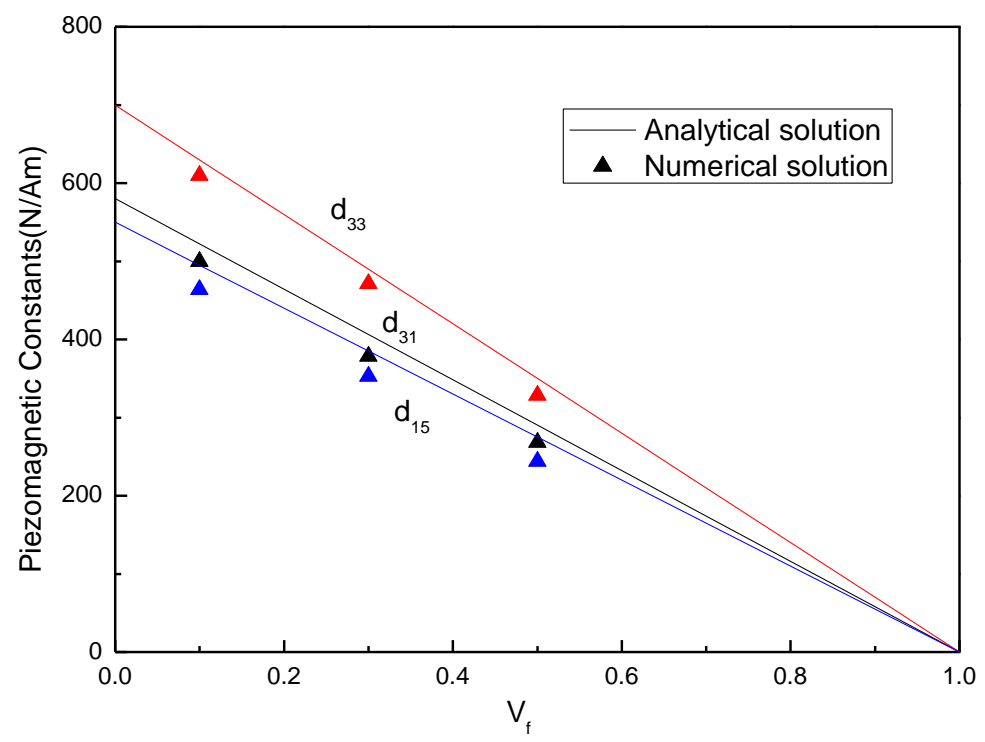

Fig. 6 The effects of volume fraction on the effective piezomagnetic constants

\section{Conclusions}

A fast multipole BEM analysis for solving 2D problems of magneto-electro-elastic composites has been presented in this paper. A particular form of the fundamental solution, which is very appropriate for the development of FMM algorithm, is derived with the help of the Fourier transform. It is seen that the present fundamental solution is very similar to that for the $2 \mathrm{D}$ potential problem, so that it is quite easy to implement the 2D fast multipole BEM for heterogeneous MEE media. Several numerical examples are given to demonstrate the accuracy, efficiency, and versatility of the developed FMBEM. Numerical examples, including rectangular magneto-electro-elastic sheets with a single inclusion or randomly distributed multi-inclusions are investigated. The advantages of FMBEM, i.e., its higher speed of computation and lower memory requirements than conventional approaches, make it appropriate as a tool to deal with many potential application problems, such as those in smart structures and MEMS or NEMS technology.

The extension of the present fast multipole BEM formulations to 3D MEE problems will be an interesting topic. Besides, consideration of interfacial cracking process of multi-inclusion magneto-electro-elastic composites under external loadings will be another challenge, which is very important for the exploration of failure mechanism of this kind of composites. Research results along these lines will be reported in future works.

\section{Acknowledgements}

The work was supported by the National Natural Science Foundation of China (Nos. 10725210 and 10832009) and the National Basic Research Program of China (No. 2009CB623204).

\section{Appendix (A note on the integral representations of the solution)}

The integral representation of the solution in Eq.(4) has been derived from the following identity: 


$$
\begin{aligned}
& \int_{\Omega}\left[c_{i j k l} u_{k, j l}+e_{l i j} \phi_{, j}+d_{l i j} \psi_{, l j}\right] u_{i}^{*} \mathrm{~d} \Omega+\int_{\Omega}\left[e_{i k l} u_{k, l i}-\varepsilon_{i l} \phi_{, i l}-g_{i l} \psi_{, i l}\right] \phi^{*} \mathrm{~d} \Omega \\
& +\int_{\Omega}\left[d_{i k l} u_{k, l i}-g_{i l} \phi_{, i l}-\mu_{i l} \psi_{, i l}\right] \psi^{*} \mathrm{~d} \Omega=\int_{\Gamma}\left(t_{i} u_{i}^{*}-\omega \phi^{*}-\eta \psi^{*}\right) \mathrm{d} \Gamma \\
& -\int_{\Gamma} u_{k}\left(c_{i j k l} u_{i, j}^{*}+e_{i k l} \phi_{, i}^{*}+d_{i k l} \psi_{, i}^{*}\right) \mathrm{n}, \mathrm{d} \Gamma+\int_{\Gamma} \phi\left(-e_{l i j} u_{i, j}^{*}+\varepsilon_{i l} \phi_{, i}^{*}+g_{i l} \psi_{, i}^{*}\right) \mathrm{n}_{l} \mathrm{~d} \Gamma \\
& +\int_{\Gamma} \psi\left(-d_{l i j} u_{i, j}^{*}+g_{i l} \phi_{, i}^{*}+\mu_{i l} \psi_{, i}^{*}\right) \mathrm{n}_{l} \mathrm{~d} \Gamma+\int_{\Omega} u_{k}\left(c_{i j k l} u_{i, j l}^{*}+e_{i k l} \phi_{, i l}^{*}+d_{i k l} \psi_{, i l}^{*}\right) \mathrm{d} \Omega \\
& -\int_{\Omega} \phi\left(-e_{l i j} u_{i, j l}^{*}+\varepsilon_{i l} \phi_{, i l}^{*}+g_{i l} \psi_{, i l}^{*}\right) \mathrm{d} \Omega-\int_{\Omega} \psi\left(-d_{l i j} u_{i, j l}^{*}+g_{i l} \phi_{, i l}^{*}+\mu_{i l} \psi_{, i l}^{*}\right) \mathrm{d} \Omega
\end{aligned}
$$

where $c_{i j k l}, e_{i k l}, d_{i k l}, \varepsilon_{i l}, g_{i l}$ and $\mu_{i l}$ are the elastic, piezoelectric, piezomagnetic, dielectric, electromagnetic and magnetic constants, respectively. Functions with superposed $*$ are arbitrary functions. Substituting fundamental solutions (solutions of Eq.(6) obtained by replacing $X_{i}(i=1, \ldots, 4)$ by Dirac's delta) into starred functions in Eq.(A.1), one obtains the expression in Eq.(4).

Note that the general theory which uses the fundamental solution of the adjoint system leads to the same result, but the detail is omitted.

\section{Reference}

1. Pan E. Exact solution for simply supported and multilayered magneto-electro-elastic plates. J Appl Mech 2001; 68: 608-618.

2. Pan E., Heylinger PR. Free vibration of simply supported and multilayered magneto-electro-elastic plates. J Sound Vibrat 2002; 253(3): 429-442.

3. Pan E. Three dimensional Green's function in anisotropic magneto-electro-elastic bimaterials. Z Angew Math Phys 2002; 53: 815-838.

4. Wang X, Shen YP. The general solution of three-dimensional problems in magneto-electro-elastic media. Int J Eng Sci 2002; 40: 1069-1680.

5. Chen WQ, Lee KY. Alternative state space formulations for magnetoelectric thermoelasticity with transverse isotropy and the application to bending analysis of nonhomogeneous plates. Int J Solids Struct 2003; 40(21): 5689-5705.

6. Chen WQ, Lee KY, Ding HJ. On free vibration of non-homogeneous transversely isotropic magneto-electro-elastic plates. J Sound Vib 2005; 279(1-2): 237-251.

7. Chen WQ, Lee KY, Ding HJ. General solution for transversely isotropic magneto-electro-thermo-elasticity and the potential theory method. Int J Eng Sci 2004; 42(13-14): 1361-1379.

8. Milazzo A, Orlando C, Alaimo A. An analytical solution for the magneto-electro-elastic bimorph beam forced vibrations problem. Smart Mater Struct 2009; 18: 085012.

9. Buchanan G R. Layered versus multiphase magneto-electro-elastic composites. Composites B 2004; 35: 413-420.

10. Pettermann HE and Suresh S. A comprehensive unit cell model: a study of coupled effects in piezoelectric 1-3 composites. Int J Solids and Struct 2000; 37: 5447-5464.

11. Lee J, Boyd JG, Lagoudas DC. Three-phase electro-magneto-elastic multifunctional composite materials, in: SPIE $10^{\text {th }}$ Annual International Symposium on Smart Structures held at San Diego, CA, March 2-6, V5053. 2003. 
12. Ding HJ, Jiang AM. A boundary integral formulation and solution for $2 \mathrm{D}$ problems in magneto-electro-elastic media. Compos Struct 2004; 82: 1599-1607.

13. Ding HJ, Jiang AM, Hou PF, Chen WQ. Green's functions for two-phase transversely isotropic magneto-electro-elastic media. Eng Anal Bound Elem 2005; 29: 551-561.

14. Davi G, Milazzo A and Orlando C. Magneto-electro-elastic bimorph analysis by the boundary element method. Mechanics of Advance Materials and Structures 2008; 15: 220-227.

15. Nishimura N. Fast multipole accelerated boundary integral equation methods. Applied Mechanics Reviews 2002; 55(4): 299-325.

16. Sabariego RV, Gyselinck J, Geuzaine C, Dular P and Legros W. Application of the fast multipole method to the 2D finite element-boundary element analysis of electromechanical devices. Compel 2003; 22(3): 659-673.

17. Hou PF, Ding HJ, Chen JY. Green's functions for transversely isotropic magnetoelectroelastic media. Int J Eng Sci 2005; 43: 826-858.

18. Liu YJ, Nishimura N. The fast multipole boundary element method for potential problems: A tutorial. Eng Anal Bound Elem 2006; 30: 371-381.

19. Sih GC, Yu HY. Volume fraction effect of magnetoelectroelastic composite on enhancement and impediment of crack growth. Compos Struct 2005; 68: 1-11.

20. Song ZF, Sih GC. Electromechanical influence of crack velocity at bifurcation for poled ferroelectric materials. Theor Appl Fract Mech 2002; 38(2): 121-39. 